\title{
Orientaciones para el manejo de la especie forestal Jigua Negro (Ocotea cernua) a partir de resultados de investigación sobre su estructura poblacional y estado de desarrollo en el departamento del Chocó
}

\author{
Guidelines for the management of forest species Jigua Black \\ (Ocotea cernua) based on results of research into their population \\ structure and state of development in department of Chocó
}

\section{William Klinger Brahan*}

\section{Resumen}

El presente artículo surge del resultado de una investigación realizada por el Instituto de Investigaciones Ambientales del Pacífico (IIAP) con el auspicio de la Corporación Autónoma para el Desarrollo Sostenible del Chocó (CODECHOCO), dirigida a conocer el estado de conservación de varias especies forestales reportadas en condición de amenaza por la presión antrópica ejercida sobre ellas. En el estudio se investigó la estructura poblacional y el estado de desarrollo de la especie forestal Jigua Negro (Ocotea cernua), encontrando que en relación con las otras especies estudiadas, su frecuencia, abundancia e importancia en las áreas muestreadas es muy alta, lo cual seguramente se debe a la baja cultura de uso local que tiene su madera en el departamento del Chocó. A pesar de lo anterior, al analizar el estado de desarrollo de las poblaciones encontradas, sus individuos muestran casi un confinamiento o restricción a las clases diamétricas inferiores, lo que sin duda impide que su gran abundancia se traduzca en verdaderas opciones de aprovechamiento sostenido en el tiempo, razón por la cual se proponen estrategias de manejo orientadas a su conservación, lo cual supone su uso sostenible.

Palabras clave: Jigua negro; Especies forestales; Manejo de Ocotea cernua.

\begin{abstract}
This article comes from the result of an investigation by the Instituto de Investigaciones Ambientales del Pacifico (IIAP) sponsored by the Corporación Autónoma para el Desarrollo Sostenible del Chocó (CODECHOCO) directed to meet the conservation status of several forest species reported in threat condition by anthropic pressure exerted on them. The study investigated the population structure and state of development of the forest species Jigua Black (Ocotea cernua), finding that in relation to the other species studied, frequency, abundance and importance in the areas sampled is very high, which is probably due to low local culture of use that has its wood in the department of Choco. Despite this, when analyzing the state of development of the populations found, its individuals show almost a confinement or restriction on the lower diameter classes, which certainly keeps their abundance translates into real sustainable use options in the time, why are proposed management strategies aimed at conservation, which means its sustainable use.
\end{abstract}

Keywords: Jigua black; Forest species; Ocotea cernua of management.
* Ingeniero Forestal, Profesor Titular, Universidad Distrital Francisco José de Caldas, Bogotá, y Director General del Instituto de Investigaciones Ambientales del Pacífico (IIAP), Quidbó, Colombia. e-mail: wklinger@iiap.org.co Recibido: 5 de enero de 2012 Aceptado: 30 de marzo de 2012 


\section{Introducción}

Estudios recientes desarrollados por el Instituto de Investigaciones Ambientales del Pacífico (IIAP) con el patrocinio del Ministerio de Ambiente y Desarrollo Sostenible (MADS), sobre aprovechamiento y movilización de maderas, el cobro de tasas compensatorias y su relación con los instrumentos de planificación forestal que existen en Colombia, muestran en términos económicos, la importancia de esta actividad productiva para los habitantes de la región y exige una mirada propositiva dirigida a mantener la oferta de recursos forestales como una posibilidad de generación de ingresos de manera sostenida en el departamento del Chocó.

Desde la perspectiva anterior, se trata de aprovechar los resultados de un trabajo de investigación recientemente desarrollado (IIAP, MADS 2012), que tuvo como propósito conocer el estado de las poblaciones de varias especies forestales reportadas en amenaza, en la que se encontró una altísima frecuencia y abundancia relativa del Jigua Negro (Ocotea cernua), desde lo cual se percibe la necesidad de orientar su manejo para promover su aprovechamiento sostenible.

A pesar que se encontró un alto número de individuos de la especie durante el estudio, la distribución diamétrica de la población muestra que los árboles no alcanzan un estado de desarrollo óptimo, que se rezagan quedando en las clases diamétricas inferiores e intermedias, razón por la cual se proponen orientaciones de manejo dirigidas a garantiza el uso sostenible de la madera de esta especie, lo cual supone la promoción de los especímenes hacia las clases diamétricas superiores, de tal manera que se incrementen los volúmenes aprovechables y al mismo tiempo se reduzca la presión sobre otras especies forestales que muestran poblaciones mucho más reducidas.

El presente estudio buscó definir orientaciones de manejo para el aprovechamiento sostenible de la madera de Jigua Negro (Ocotea cernua), a partir del conocimiento de diferentes parámetros de su estructura poblacional y su estado de desarrollo en cinco municipios del departamento del Chocó. Para ello se estableció la frecuencia y densidad poblacional de las especie, se determinó el estado de desarrollo y la estructura diamétrica de los individuos, lo que permitió además identificar estrategias de manejo de la especie Jigua Negro orientadas a la promoción del aprovechamiento sostenible de su madera.

\section{Información general de la especie}

Nombre común: Jigua Negro

Familia: Lauraceae

Nombre científico: Ocotea cernua
Aspectos sobresalientes del árbol. Mide de 5 a $20 \mathrm{~m}$ de altura y de $10 \mathrm{a} 50 \mathrm{~cm}$ de diámetro. Posee una copa umbelada o redondeada. Tronco ramificado a baja altura, a veces con rebrotes en la base. Corteza exterior negra. Ramitas terminales verdes y glabras. Hojas simples y alternas, aromáticas al estrujarlas, de 5 a $15 \mathrm{~cm}$ de largo y de 3 a $5 \mathrm{~cm}$ de ancho, elípticas a oblongo-elípticas, con ápice acuminado, bordes ondulados y base obtusa. Pecíolos de 1 a $2 \mathrm{~cm}$ de largo. La especie es dioica. Flores verdes o amarillentas, aromáticas, a veces con un ligero olor a limón. Frutos en drupas de 1 a 1.5 $\mathrm{cm}$ de largo y con una copa basal de color rojizo, verdes, tornándose negros al madurar.

Datos ecológicos. La especie crece a bajas y medianas elevaciones, en climas húmedos o muy húmedos. Común y ampliamente distribuida en los bosques del canal de Panamá. Florece y fructifica de febrero a septiembre. Las flores son visitadas por abejas y otros insectos. Las semillas son dispersadas por animales.

Especies parecidas. Por el parecido de las hojas se puede confundir con Lacistema aggregatum, pero en estas las hojas tienen los bordes dentados y los frutos son cápsulas, tornándose rojos y dehiscentes al madurar, lo cual no ocurre en $O$. cernua. También puede confundirse con Ocotea arcuata, pero sus las hojas tienen las nervaduras secundarias muy reticuladas y onduladas.

Usos. Madera empleada en la construcción de puentes y pisos industriales (Baluarte, 2011).

\section{Aspectos metodológicos}

Se escogieron cinco municipios del departamento del Chocó para la realización de los muestreos (Riosucio, Carmen del Darién, Istmina, Juradó y Río Quito), lo cual obedeció a criterios de reporte comunitario de existencias de la especie y también a la decisión de muestrear áreas con condiciones ecológicas disímiles en la región.

En cada uno de los municipios se estableció un número diferencial de parcelas hasta sumar 13.49 hectáreas. Las parcelas se diseñaron de $10 \mathrm{~m}$ x $10 \mathrm{~m}$ y en cada uno de ellas se registraron y se hizo ubicación geográfica a todos los individuos que se encontraron de la especie. En la Tabla 1 se detalla el número de parcelas utilizadas para el muestreo en cada uno de los municipios escogidos.

Todos los individuos se registraron en un formato prediseñado que incluyó información sobre diámetro, altura comercial y altura total, correspondientes a variables de tipo cuantitativo; de igual manera se registró información sobre la forma y tamaño de las copas, presencia de bejucos y estado fitosanitario, variables de tipo cualitativo que presentan relevancia en los diferentes estados del bosque. La Figura 1 muestra detalles del registro de la información en campo. 
Bioetnia Volumen 9 № 1 (enero-junio), 2012

Tabla 1

Número de parcelas de $10 \mathrm{~m} \times 10 \mathrm{~m}$ muestreadas por sitio

\begin{tabular}{lc}
\hline Sitio de muestreo & Número de parcelas \\
\hline Istmina & 205 \\
Río Quito & 196 \\
Riosucio & 226 \\
Carmen del Darién & 62 \\
Juradó & 660 \\
\hline Total & 1.349 \\
\hline
\end{tabular}

Se consideró pertinente la colección de material vegetal para la identificación de las especies, éstas fueron entregadas al herbario de la Universidad Tecnológica del Chocó, estamento que realizó la identificación hasta los niveles que se encuentran reportados en este documento. La Figura 2 muestra los detalles de la recolección y preparación de muestras botánicas.

La compilación y tabulación de los datos registrados se realizó mediante la utilización de base de datos en Office Excel tomando como referente los cuadros y fórmulas establecidas en la guía técnica del Ministerio de Ambiente y Desarrollo Sostenible (2002), las cuales permitieron generar tablas y gráficas que facilitaron el análisis de la información.

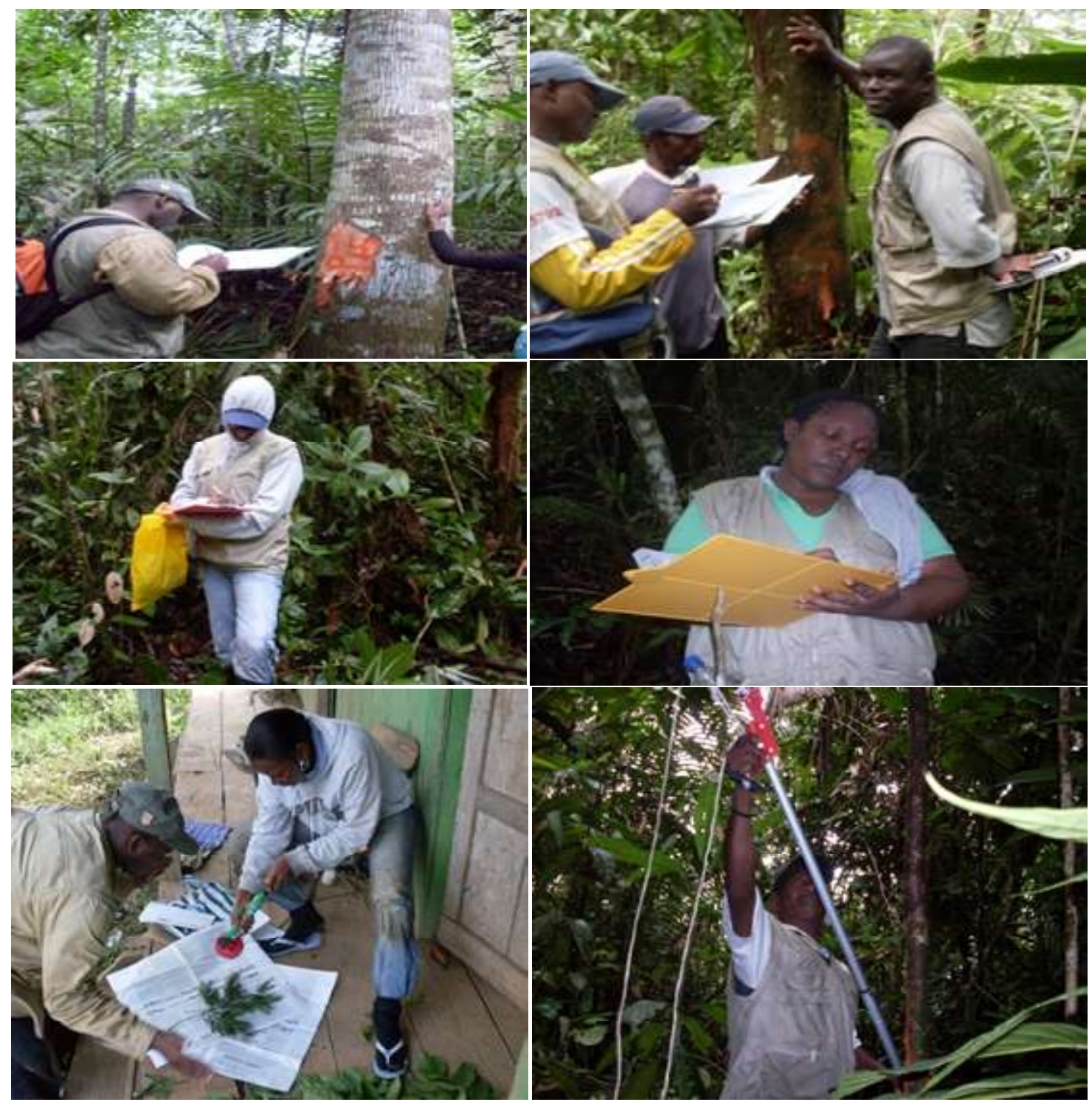

Figura 1. Registro de la información en campo 


\section{Resultados y discusión}

Análisis de frecuencia y densidad poblacional de la especie. Los datos de campo muestran tanto frecuencia como densidad poblacional relativamente alta para la especie. La media de la frecuencia calculada para los cinco municipios donde se realizó el muestreo alcanza un valor superior al $76 \%$, en tanto que la densidad poblacional supera los 211 individuos por hectárea, lo cual resulta ser sin duda significativo frente a datos conocidos para otras especies en los mismos sitios de muestreo.

Como referencia, utilizando los mismos sitios de muestreo y las mismas superficies muestreadas, se tienen datos de densidades poblacionales en número de individuos por hectárea, del Abarco (Cariniana pyriformis) 84.73, del Pino Amarillo (Podocarpus sp) 3.48, del Guayacán Amarillo (Tabebuya crysantha) 3.33 y del Guayaquil (Centrolobium paraense) 1.04 , lo cual muestra una densidad poblacional muy superior del Jigua Negro en comparación con otras especies forestales de amplio valor de uso en el departamento del Chocó, e indica al mismo tiempo, la escasez a las que éstas podrían verse avocadas en el futuro cercano.

En cuanto a frecuencia ocurre una situación similar, mientras que en un total 1.349 parcelas de $10 \mathrm{~m}$ x $10 \mathrm{~m}$ montadas durante el estudio, la especie Jigua Negro hace presencia en 904, el Abarco lo hace en 449, el Pino Amarillo en 19 y el Guayaquil y el Guayacán Amarillo en 6. Estos datos muestran una notable superioridad de frecuencia del Jigua Negro, aproximadamente 2 veces más que el Abarco, 48 veces más que el Pino Amarillo y 151 veces más que el Guayaquil y el Guayacán Amarillo. La Tabla 2 muestra información detallada sobre el tema.

Ahora bien, haciendo un análisis comparativo de los datos entre municipios, Istmina presenta la mayor densidad de Jigua Negro en número de individuos por unidad de superficie, dato que permite inferir que hay abundancia de la

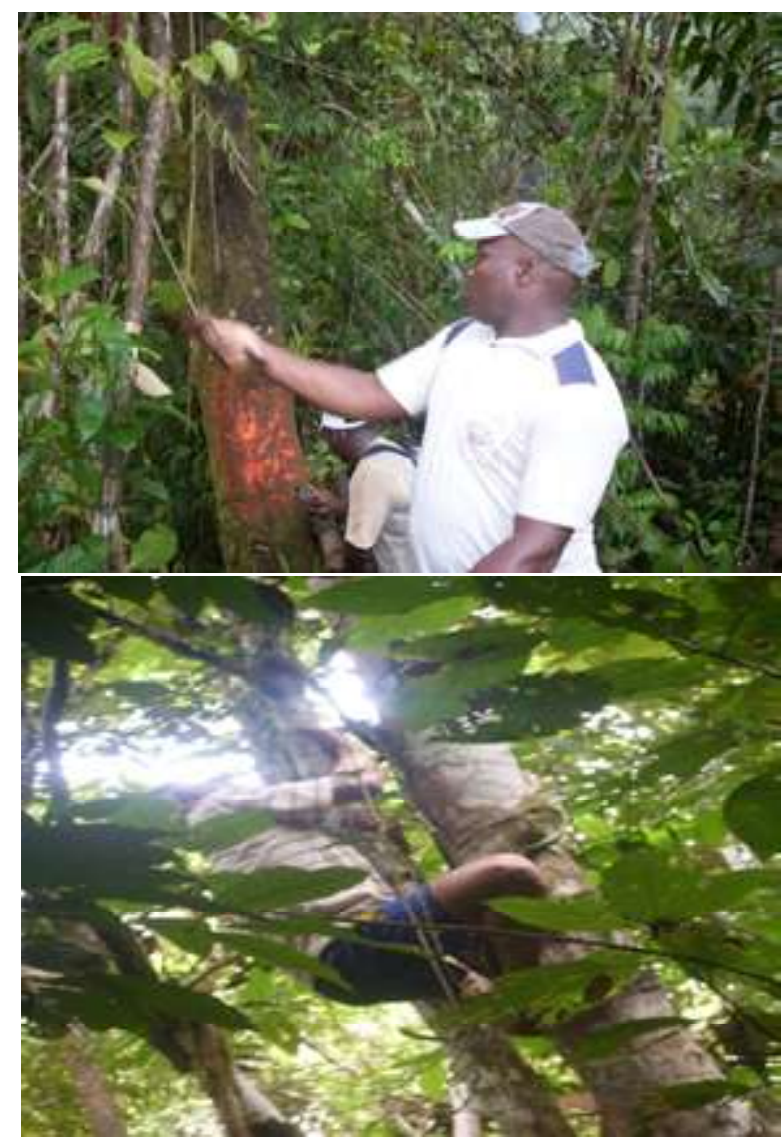

Figura 2. Recolección y preparación de muestras botánicas

especie en la Cuenca del San Juan y en condiciones climáticas de mayor precipitación. Obsérvese que los municipios ubicados más hacia el norte del Chocó, Riosucio en la Cuenca del Atrato y Juradó en la Cuenca del Pacífico, presentan los más bajos niveles de densidad poblacional de la especie, y tienen

Tabla 2

Número de individuos de Jigua Negro por sitio de muestreo en cinco municipios del Chocó

\begin{tabular}{lcccc}
\hline Municipio & \multicolumn{4}{c}{ Número } \\
\cline { 2 - 5 } & $\begin{array}{c}\text { de parcelas en el que } \\
\text { se encontró la especie }\end{array}$ & $\begin{array}{c}\text { de parcelas de } \\
\mathbf{1 0} \mathbf{~} \mathbf{~ x ~ 1 0 ~} \mathbf{~ m}\end{array}$ & $\begin{array}{c}\text { total de individuos } \\
\text { encontrados }\end{array}$ & $\begin{array}{c}\text { de árboles } \\
\text { por hectárea }\end{array}$ \\
\hline Carmen del Darién & 96.77 & 62 & 164 & 264.50 \\
\hline Istmina & 88.78 & 205 & 658 & 320.97 \\
\hline Juradó & 55.45 & 660 & 1.371 & 207.72 \\
\hline Río Quito & 84.18 & 196 & 416 & 212.24 \\
\hline Riosucio & 57.96 & 226 & 247 & 109.29 \\
\hline Total & 76.73 & 1.349 & 2.856 & \\
\hline
\end{tabular}




\section{Bioetnia Volumen 9 № 1 (enero-junio), 2012}

relativos bajos niveles de precipitación. De la misma manera, en estos sitios la frecuencia de la especie es más baja que en los otros tres municipios donde se realizaron los muestreos, mientras en Riosucio y Jurado la frecuencia de la especie no alcanza el 60\%, en los otros municipios, Carmen del Darién, Río Quito e Istmina tienen una frecuencia de Jigua Negro cercana o superior al $85 \%$.

Análisis del estado de desarrollo de la especie. Durante el muestreo se registraron en total 2.856 individuos de la especie forestal Jigua Negro, $1.737(60.8 \%)$ en estado de brinzal, 532 (18.6\%) en estado de latizal y 587 (20.6\%) en estado de fustal, lo que en términos generales corresponde a una distribución normal de los individuos que confinan su mayor cantidad en la regeneración natural, que luego va reduciendo el número de individuos en la medida en que avanza hacia estadíos de desarrollo superiores. Sin embargo, es evidentemente notoria la ruptura de esta regularidad en el caso del municipio de Carmen del Darién; en este sitio la regeneración natural es escasa y la mayor parte de individuos se encuentra en estado de fustal (75.6\%), lo que puede ser indicativo de un bosque que está cerrando o tiene cerrado su dosel, con lo que se restringe el paso de luz al sotobosque y con ello de igual manera se reducen las posibilidades de germinación de semillas o nacimiento de renuevos.

Una situación similar se presenta en el municipio de Riosucio donde el mayor porcentaje de individuos de la especie Jigua Negro se encuentran en estado de fustal (72.5\%). Significa lo anterior, que hay una baja cultura de uso de la madera de Jigua Negro en estos municipios, los cuales se encuentran próximos o contiguos, lo que hace que los parches de bosque donde se encuentra la especie no sufran presión por deforestación.

En el municipio de Juradó ocurre lo contrario, se presenta una abundante regeneración natural con más del $80 \%$ de la población de la especie en estado de brinzal y latizal, lo que está mostrando una dificultad para alcanzar la condición de fustal, sin que se tenga claridad sobre las causas que pueden originar este comportamiento; por una parte puede ser que fustales de la especie con bajo diámetro, se vean perjudicados por el sistema de aprovechamiento de otras especies de mayor importancia comercial que en el proceso de tumba, troceo y transporte menor originan daños y pérdida de material vegetal, y por otra parte, que los mecanismos de selección natural de las especies que hay en el bosque vayan imponiendo la condición existente.

En los municipios de Río Quito e Istmina, parece presentarse una situación normal, sin exageraciones de cantidad, la mayor parte de los individuos de la especie Jigua Negro se encuentran en estado de brinzal y latizal, lo que muestra regularidad en la distribución por estado de desarrollo, también porque a pesar de ser una menor cantidad, existe un número de individuos considerables en condición de fustal.
La Tabla 3 muestra información detallada sobre el estado de desarrollo de la especie en los cinco municipios del Chocó que se muestrearon.

\section{Tabla 3}

\section{Distribución de individuos de Jigua Negro por estado de desarrollo en cinco municipios de Chocó}

\begin{tabular}{lrrrr}
\hline Municipio & Brinza & Latizal & Fustal & Total \\
\hline Carmen del Darién & 18 & 22 & 124 & 164 \\
Istmina & 381 & 209 & 68 & 658 \\
Juradó & 1079 & 172 & 120 & 1371 \\
Río Quito & 219 & 101 & 96 & 416 \\
Riosucio & 40 & 28 & 179 & 247 \\
Total & 1.737 & 532 & 587 & 2.856 \\
\hline
\end{tabular}

Análisis de la distribución diamétrica de la especie. La distribución de los individuos por clase diamétrica muestra una gran regularidad de la especie Jigua Negro, excepto la existencia de un notorio número mayor (4 veces) de individuos con diámetros superiores a los $60 \mathrm{~cm}$ en relación con la clase diamétrica inmediatamente inferior $(50$ a $60 \mathrm{~cm}$ de diámetro), y el bajo número de individuos en esta categoría en relación con el elevado número de individuos en la categoría de $\operatorname{los} 0$ a los $10 \mathrm{~cm}$ de diámetro, 20 y 2.246 respectivamente.

Lo anterior muestra dos elementos importantes al momento de la definición de orientaciones de manejo; por una parte, la necesidad de impulsar el uso local o nacional de la madera de la especie Jigua Negro, la cual no aparece en los registros de solicitud de aprovechamiento de los últimos años, lo que puede explicar la existencia de más de 11 individuos por hectárea con diámetros iguales o superiores a los $40 \mathrm{~cm}$ en el área de estudio; por otra parte, la necesidad de promover las condiciones para que más individuos alcancen las clases diamétricas superiores, dada la gran abundancia de renuevos de la especie; más de 179 individuos por hectárea con diámetros iguales o inferiores a $20 \mathrm{~cm}$ y más de 162 individuos por hectárea con diámetros iguales o inferiores a $10 \mathrm{~cm}$.

El 78.6\% de los individuos de Jigua Negro inventariados se encuentran en la primera clase diamétrica que va hasta los $10 \mathrm{~cm}$ de diámetro, $8.1 \%$ está en la segunda clase diamétrica, la cual va de 10 a $20 \mathrm{~cm}, 5.5 \%$ conforma la tercera clase diamétrica que comprende los individuos entre 20 y 30 centímetros de diámetro, en la cuarta clase diamétrica (30 a $40 \mathrm{~cm}$ ) se encuentra $2.7 \%$ de los individuos, 40 individuos que corresponden al 1.4\% están en la quinta clase diamétrica que va de los 40 a $\operatorname{los} 50 \mathrm{~cm}, 0.7 \%$ de los individuos se 
encuentra en la sexta clase diamétrica $(50$ a $60 \mathrm{~cm})$, y finalmente, $2.9 \%$ de los individuos se registraron con diámetros superiores a $60 \mathrm{~cm}$.

Los datos anteriores, tal como se dijo antes, muestran una gran regularidad en la distribución general de los individuos de la especie Jigua Negro por clases diamétricas, asemejándose el gráfico de esta distribución a una jota invertida, reportada por muchos autores para indicar el comportamiento normal de una especie, con las excepciones que se mencionaron antes. En la Tabla 4 se presenta información detallada sobre la estructura de la especie.

El análisis comparativo entre municipios muestra particularidades muy especiales que se deben tomar en cuenta al momento de definir criterios generales de manejo para la especie en el departamento del Chocó.

En el municipio de Juradó una abundantísima regeneración natural, aproximadamente $86 \%$ de la población de la especie en la clase diamétrica inferior (1.178 individuos), 30 individuos entre los 30 y los $60 \mathrm{~cm}$ de diámetro, lo que acusa la necesidad de dar un mejor manejo a la regeneración natural para promover el paso de más individuos a estadíos de desarrollo superiores. También se destaca la presencia de 76 individuos con diámetros mayores a $60 \mathrm{~cm}$, lo que da cuenta de una baja cultura de uso local de la especie. Buena parte de las excepciones que en general se encuentran al comportamiento regular de la especie según la distribución de sus especímenes por diámetro, se explican por lo que ocurre en el municipio de Juradó, el cual posee $48 \%$ de la población total de Jigua Negro encontrada en los sitios de muestreo.

En el municipio de Istmina los datos muestran un alto porcentaje de individuos en la primera clase diamétrica (96.6\%) y sólo 2 de 648 individuos en las últimas tres clases diamétricas superiores, lo que indica sin duda una gran dificultad de la especie Jigua Negro para avanzar hacia estados de desarrollo que hagan más aprovechables sus individuos desde el punto de vista maderero. En consecuencia se requiere un estudio detallado que investigue de manera exhaustiva las causas que originan esta situación de la especie particularmente en esta municipalidad que se utilizó como sitio de muestreo.

En los municipios de Riosucio y Carmen del Darién los datos encontrados muestran una distribución muy normal de los individuos de Jigua Negro conforme a sus diámetros, aunque con algunas irregularidades hay una concentración normal de la mayor cantidad de individuos en las clases diamétricas inferiores, la cual se va reduciendo en la medida en que se avanza hacia las clases diamétricas superiores; la tendencia de los datos es a formar una jota invertida, sin que sean sustanciales las anormalidades que se presentan, es posible que este comportamiento pueda atribuirse a la mayor vocación y cultura de aprovechamiento forestal que hay en estas tierras, lo que hace normal la actividad productiva y en consecuencia el comportamiento del bosque.

En el municipio de Río Quito aun cuando hay una tendencia a la normalidad, también existe una irregularidad pronunciada, y es el alto porcentaje de individuos en la primera clase diamétrica; de los 658 individuos de Jigua Negro que se encuentran en total en este sitio de muestreo, 626 tienen diámetros iguales o inferiores a $10 \mathrm{~cm}$, lo que corresponde a un porcentaje de $95.1 \%$, situación que indica que estos no están avanzando de manera regular hacia las clases diamétricas superiores, así mantengan la tendencia de tener un bajo número de individuos en ellas.

Análisis de la abundancia y la dominancia de la especie. Antes de adentrarse en este análisis cabe resaltar que la abundancia y dominancia del Jigua Negro en cada uno de los cinco municipios que conforman elárea de estudio, se realizó en comparación con otras especies forestales que pueden resultar ligeramente distintas para cada sitio.

En el municipio de Carmen del Darién, el Jigua Negro resulta ser bastante más abundante que otras especies forestales como el Abarco, Carrá, Choibá y Pino Amarillo. Teniendo la misma área de referencia, se encuentran 164 individuos de Jigua Negro y sólo 17 de Abarco, 7 de Carrá,

Tabla 4

Distribución de individuos de Jigua Negro por clase diamétrica en cinco municipios del Chocó

\begin{tabular}{|c|c|c|c|c|c|c|c|c|}
\hline \multirow[t]{2}{*}{ Municipio } & \multicolumn{7}{|c|}{ Clase diamétrica $(\mathrm{cm})$} & \multirow[t]{2}{*}{ Total } \\
\hline & $0-10$ & $10-20$ & $20-30$ & $30-40$ & 40-50 & $50-60$ & $>60$ & \\
\hline Carmen del Darién & 40 & 58 & 38 & 16 & 6 & 3 & 3 & 164 \\
\hline Istmina & 626 & 17 & 8 & 5 & & 2 & & 658 \\
\hline Juradó & 1.178 & 59 & 28 & 6 & 14 & 10 & 76 & 1.371 \\
\hline Río Quito & 334 & 52 & 20 & 5 & 3 & 2 & & 416 \\
\hline Riosucio & 68 & 46 & 64 & 45 & 17 & 3 & 4 & 247 \\
\hline Total & 2.246 & 232 & 158 & 77 & 40 & 20 & 83 & 2.856 \\
\hline
\end{tabular}


29 de Choibá y 3 de Pino Amarillo, una abundancia relativa muy superior del Jigua Negro en comparación con cada una de las otras especies forestales. A pesar de lo anterior, la dominancia del Jigua Negro resulta inferior a la del Abarco y la del Choibá, dada la mayor existencia de árboles de estas especies en clases diamétricas superiores, razón por la que aportan una mayor área basal que las hace dominantes. En la Tabla 5 se presenta información al respecto de la abundancia y dominancia de la especie.

En el municipio de Istmina el Jigua Negro es más abundante que el Guayacán Amarillo, el Chanó y el Níspero y que el Guayacán Negro, es decir, que todas las especies estudiadas al mismo tiempo. De igual manera, es la más dominante, su aporte de casi $3 \mathrm{~m}^{2}$ de área basal así lo define. En la Tabla 6 se detalla información que incluye tanto el número de árboles encontrados para cada especie, como el área basal que cada una de ellas presentó, datos con los que se puede calcular abundancia y dominancia, para así determinar la gran importancia que tiene la especie forestal Jigua Negro en el municipio chocoano de Istmina, utilizando como sitios de muestreo Santa Mónica y Puerto Salazar.
En el municipio de Juradó el comportamiento del Jigua Negro sigue la misma tendencia que en los otros municipios; a pesar que los parámetros en estudio se determinaron tomando en cuenta un mayor número de especies forestales. La abundancia y dominancia del Jigua Negro es más alta que la del Abarco, Guayaquil, Guayacán Amarillo, Pino Amari1lo, Cedro, Chanó, Caoba y Roble, lo cual se explica por el alto número de individuos que se encontraron de Jigua Negro en comparación con estas otras especies forestales, y por la gran diferencia de área basal que esta presenta (Tabla 7).En Río Quito, también es el Jigua Negro la especie más abundante y dominante, su aporte de más del $66 \%$ de los individuos que en total se encontraron y de aproximadamente $40 \%$ del área basal total del bosque analizado, así lo establecen. Las otras especies estudiadas son Abarco, Algarrobo, Chanó, Chachajo, Trúntago, Incibe y Guayacán Amarillo (Tabla 8).

Riosucio es el único de los cinco municipios en el que el Jigua Negro no es la especie más abundante y dominante, en estos aspectos es superado por el Abarco, especie de la que se encontraron 768 de un total de 1.046 individuos y un área basal de $24 \mathrm{~m}^{2}$ de un total de $48 \mathrm{~m}^{2}$, las otras especies

Tabla 5

Abundancia y dominancia del Jigua Negro en comparación con otras especies forestales en Carmen del Darién, Chocó

\begin{tabular}{lccccccc}
\hline Nombre regional & Número de árboles & Área basal & \multicolumn{2}{c}{ Abundancia } & & \multicolumn{2}{c}{ Dominancia } \\
\cline { 7 - 8 } \cline { 7 - 7 } & & & Absoluta & Relativa & & Absoluta & Relativa \\
\hline Abarco & 17 & 6,841 & 17 & 7,7 & 6,8 & 11,7 \\
\hline Carrá & 7 & 5,961 & 7 & 3,2 & 6,0 & 10,2 \\
Choibá & 29 & 38,00 & 29 & 13,2 & & 38,0 & 65,3 \\
Jigua Negro & 164 & 6,50 & 164 & 74,6 & & 6,5 & 11,2 \\
\hline Pino Amarillo & 3 & 0,93 & 3 & 1,4 & & 0,9 & 1,6 \\
\hline Total & 220 & 58,232 & 220 & 100,0 & 58,2 & 100,0 \\
\hline
\end{tabular}

Tabla 6

Abundancia y dominancia del Jigua Negro en comparación con otras especies forestales en Istmina, Chocó

\begin{tabular}{|c|c|c|c|c|c|c|}
\hline \multirow{2}{*}{ Nombre regional } & \multirow[t]{2}{*}{ Número de árboles } & \multirow[t]{2}{*}{ Área basal } & \multicolumn{2}{|c|}{ Abundancia } & \multicolumn{2}{|c|}{ Dominancia } \\
\hline & & & Absoluta & Relativa & Absoluta & Relativa \\
\hline Jigua negro & 658 & 2,937 & 658 & 73,44 & 2,94 & 30,35 \\
\hline Guayacán Amarillo & 39 & 2,892 & 39 & 4,35 & 2,90 & 29,882 \\
\hline Chanó & 33 & 2,563 & 33 & 3,68 & 2,56 & 26,483 \\
\hline Níspero & 146 & 1,252 & 146 & 16,29 & 1,25 & 12,937 \\
\hline Guayacán Negro & 20 & 0,034 & 20 & 2,23 & 0,03 & 0,351 \\
\hline Total & 896 & 9,678 & 896 & 100,0 & 9,678 & 100,0 \\
\hline
\end{tabular}


Tabla 7

Abundancia y dominancia de la especie Jigua Negro en comparación con otras especies forestales en Juradó, Chocó

\begin{tabular}{|c|c|c|c|c|c|c|}
\hline \multirow[t]{2}{*}{ Nombre regional } & \multirow[t]{2}{*}{ Número de árboles } & \multirow[t]{2}{*}{ Área basal } & \multicolumn{2}{|c|}{ Abundancia } & \multicolumn{2}{|c|}{ Dominancia } \\
\hline & & & Absoluta & Relativa & Absoluta & Relativa \\
\hline Abarco & 333 & 53,36 & 333 & 14,60 & 53,36 & 21,49 \\
\hline Jigua Negro & 1371 & 113,51 & 1371 & 60,11 & 113,51 & 45,71 \\
\hline Guayaquil & 7 & 1,51 & 7 & 0,31 & 1,51 & 0,61 \\
\hline Guayacán Amarillo & 4 & 0,74 & 4 & 0,18 & 0,74 & 0,30 \\
\hline Pino Amarillo & 42 & 3,58 & 42 & 1,84 & 3,58 & 1,44 \\
\hline Cedro & 355 & 27,13 & 355 & 15,56 & 27,13 & 10,93 \\
\hline Chanó & 126 & 41,68 & 126 & 5,52 & 41,68 & 16,79 \\
\hline Caoba & 24 & 4,75 & 24 & 1,05 & 4,75 & 1,91 \\
\hline Roble & 19 & 2,05 & 19 & 0,83 & 2,05 & 0,83 \\
\hline Total & 2281 & 248,31 & 2281 & 100,0 & 248,31 & 100,0 \\
\hline
\end{tabular}

Tabla 8

Abundancia y dominancia de la especie Jigua Negro en comparación con otras especies forestales en Río Quito, Chocó

\begin{tabular}{|c|c|c|c|c|c|c|}
\hline \multirow{2}{*}{ Nombre regional } & \multirow[t]{2}{*}{ Número de árboles } & \multirow[t]{2}{*}{ Área basal } & \multicolumn{2}{|c|}{ Abundancia } & \multicolumn{2}{|c|}{ Dominancia } \\
\hline & & & Absoluta & Relativa & Absoluta & Relativa \\
\hline Abarco & 25 & 1,866 & 25 & 4,05 & 1,87 & 17,7 \\
\hline Algarrobo & 7 & 0,407 & 7 & 1,13 & 0,41 & 3,87 \\
\hline Chanó & 9 & 1,051 & 9 & 1,46 & 1,05 & 9,98 \\
\hline Chachajo & 7 & 0,214 & 7 & 1,13 & 0,21 & 2,03 \\
\hline Trúntago & 51 & 1,194 & 51 & 8,25 & 1,19 & 11,3 \\
\hline Incibe & 101 & 1,767 & 101 & 16,3 & 1,77 & 16,8 \\
\hline Jigua Negro & 416 & 4,011 & 416 & 67,3 & 4,01 & 38,1 \\
\hline Guayacán Amarillo & 2 & 0,019 & 2 & 0,33 & 0,02 & 0,18 \\
\hline Total & 618 & 10,529 & 618 & 100,0 & 10,5 & 100,0 \\
\hline
\end{tabular}

forestales como el Carrá, Choibá, Guayaquil y Pino Amarillo sí son notoriamente menos abundantes y dominantes que el Jigua Negro (Tabla 9)

Orientaciones de manejo para la especie. El manejo de la especie forestal Jigua Negro en el departamento del Chocó debe orientarse a aprovechar su abundancia, garantizando su uso integral y acorde con sus existencias y las propiedades de su madera y otros productos que de él se puedan derivar. El aprovechamiento de la especie debe garantizar al mismo tiempo su sostenibilidad en el tiempo, razón por la que se han de definirse y tomarse medidas para lograrlo.

Se propone adelantar un programa de investigaciones que en lo inmediato dé respuesta por los menos a las siguien- tes preguntas: ¿Cuáles son las propiedades de la madera del Jigua Negro y en concordancia con ello cuáles son sus usos potenciales más importantes? ¿Qué contenidos con posibilidades de utilización se encuentran en sus hojas, corteza, frutos y semillas? ¿Cuál es la fenología de la especie? ¿Cuáles son las causas de las dificultades que tiene su regeneración natural para pasar hacia estados de mayor desarrollo?

De igual manera se propone adelantar un proceso alianza interinstitucional que promueva una adecuada gobernanza en el territorio, que establezca reglas sobre su manejo y el uso de los recursos existentes, particularizando en la situación del Jigua Negro con un ejercicio de toma de conciencia y 
Bioetnia Volumen 9 No 1 (enero-junio), 2012

Tabla 9

Abundancia y dominancia de la especie Jigua Negro en comparación con otras especies forestales en Ríosucio, Chocó

\begin{tabular}{|c|c|c|c|c|c|c|}
\hline \multirow[t]{2}{*}{ Nombre regional } & \multirow[t]{2}{*}{ Número de árboles } & \multirow[t]{2}{*}{ Área basal } & \multicolumn{2}{|c|}{ Abundancia } & \multicolumn{2}{|c|}{ Dominancia } \\
\hline & & & Absoluta & Relativa & Absoluta & Relativa \\
\hline Abarco & 768 & 23,84 & 768 & 73,42 & 23,8 & 49,5 \\
\hline Carrá & 8 & 2,37 & 8 & 0,76 & 2,4 & 4,9 \\
\hline Choibá & 14 & 7,45 & 14 & 1,34 & 7,5 & 15,5 \\
\hline Guayaquil & 7 & 1,34 & 7 & 0,67 & 1,3 & 2,8 \\
\hline Jigua Negro & 247 & 12,81 & 247 & 23,61 & 12,8 & 26,6 \\
\hline Pino Amarillo & 2 & 0,37 & 2 & 0,19 & 0,4 & 0,8 \\
\hline Total & 1.046 & 48,175 & 1.046 & 100,0 & 48,175 & 100,0 \\
\hline
\end{tabular}

capacitación ciudadana, que haga posible el conocimiento de las potencialidades de la especie y las condiciones específicas para promover su aprovechamiento sostenible, existencias totales, turnos, diámetros mínimos de corta, intensidades de corta, etc.

\section{Conclusiones}

Los datos muestran de manera contundente la importancia en términos de estructura del bosque de la especie Jigua Negro en relación con otras especies de alta cultura de uso local en el departamento del Chocó, entre ellas, Abarco, Guayacán Amarillo, Pino Amarillo, Guayaquil, Roble, Incibe, Caoba, Guayacán Negro, Chanó y Choibá. Esta situación puede obedecer a una cultura de uso menor de su madera en relación con la de estas otras especies, lo cual posibilita encontrar abundancia de individuos y buen aporte de área basal y de volumen de madera en pie.

La frecuencia y la densidad poblacional del Jigua Negro es alta en cada uno de los cinco municipios del departamento del Chocó que conformaron el área de estudio; es bastante superior a la de varias especies forestales de importancia comercial en la región y el país. Lo anterior tiene su explicación en que la madera de la especie no tiene una alta cultura de uso local y en consecuencia no es requerida ampliamente por el mercado, lo que conduce a encontrarla por muchos sitios de muestreo.

La regeneración natural del Jigua Negro es abundante en términos generales, sin embargo localidades como Carmen del Darién y Riosucio presentan bajos niveles de la misma, lo que conduce a la recomendación de un cuidadoso manejo de la zona para generar las condiciones que la favorezcan, de lo contrario, dentro de poco no habrá material vegetal para consolidar el aprovechamiento sostenible de la madera de la especie; en este sentido se deben identificar árboles semille- ros de entre los fustales que a diferencia de los brinzales y latizales, abundan.

En este mismo aspecto, la situación del Jigua Negro en el municipio de Juradó es contraria, existe una abundantísima regeneración natural que no concluye con individuos en estado de fustal en buen número, a pesar que se encuentran un buen número de árboles en condición de adultos, la existencia de regeneración natural o de individuos en brinzales y latizales es 10 veces mayor, por lo que se requiere promover el paso de un mayor número de individuos a condición de adultez, lo cual se logra con el manejo de liberaciones y entresacas que permitan el logro de estos propósitos.

El comportamiento de la especie en Istmina y Río Quito en cuanto a la distribución de individuos conforme a su estado de desarrollo tiene una tendencia mucho más normal, y en consecuencia, su manejo se debe orientar a mantener esa regularidad. La proporción entre individuos en brinzales, latizales y fustales es absolutamente ajustada a una perspectiva de aprovechamiento sostenido en el tiempo, por lo que hay que garantizar el mantenimiento de esas condiciones.

La distribución de los individuos de la especie Jigua Negro por clases diamétricas arroja información absolutamente contundentes, hay escasez de especímenes con diámetros mínimos de corta; si este se supone mayor o igual a $40 \mathrm{~cm}$ a la altura del pecho, situación que obliga a la toma inmediata de decisiones por parte de la autoridad ambiental; en los municipios de Carmen del Darién, Istmina, Río Quito y Riosucio no se debe permitir el aprovechamiento de la especie, porque el bajo número de individuos en las clases diamétricas superiores obliga a su protección para garantizar la fuente de material vegetal para mantener la buena regeneración natural de la especie.

En contraposición con lo anterior, en el municipio de Juradó sí es prudente facilitar el aprovechamiento de los árboles muy adultos, los cuales presentan diámetros a la 
altura del pecho superiores a los 60 centímetros, obviamente procurando el cuidado de proteger árboles semilleros, y con buenas características fenotípicas. Estos árboles podrían arrojar un buen volumen de madera con utilidades que podrían reinvertirse en otros proyectos productivos, que le bajen la presión al bosque por unos cuantos años; el aprovechamiento generaría la apertura de claros que le daría paso a la germinación de semillas y a una dinámica sucesional que el bosque requiere para mantenerse productivo.

La abundancia y dominancia del Jigua Negro es indiscutible, pero los datos deben mirarse con detenimiento para que no generen decisiones erradas, definitivamente existen inconvenientes para se produzca sin tropiezos y sin dificultades el paso de los individuos de clases diamétricas inferiores a superiores, lo que sin duda obliga a la implementación de un plan de manejo con énfasis en la investigación, la capacitación, la gobernanza y la liberación de competidores dentro del bosque.

\section{Literatura citada}

Bajuarte, J. 2011. Modelización del crecimiento de quince especies forestales comerciales del bosque aluvial inundable de la Amazonía peruana. Tesis doctoral. Universidad de Santiago de Compostela. $231 \mathrm{pp}$.

Instituto de Investigaciones Ambientales del Pacífico (IIAP), Ministerio de Ambiente y Desarrollo Sostenible. 2012. Formulación de una propuesta de tasa compensatoria para la conservación y el uso sostenible del recurso forestal en Colombia. Informe final. Quibdó, Bogotá; IIAP, MADS. $156 \mathrm{pp}$ 